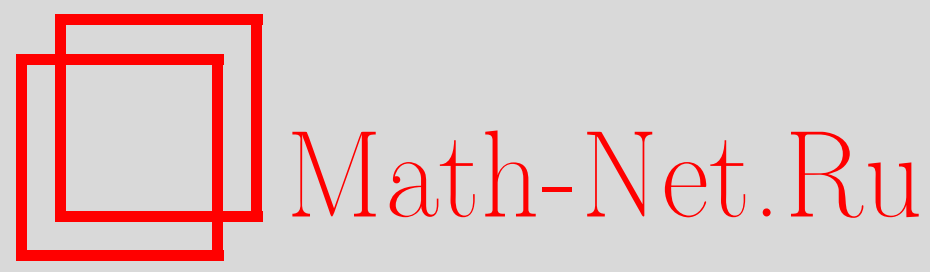

Е. А. Ровба, Сумматорные рациональные операторы типа Джексона, Матем. заметки, 1997, том 61, выпуск 2, 270277

DOI: https://doi.org/10.4213/mzm1500

Использование Общероссийского математического портала Math-Net.Ru подразумевает, что вы прочитали и согласны с пользовательским соглашением http://www. mathnet.ru/rus/agreement

Параметры загрузки:

IP : 18.207.199.55

26 апреля 2023 г., 16:20:04

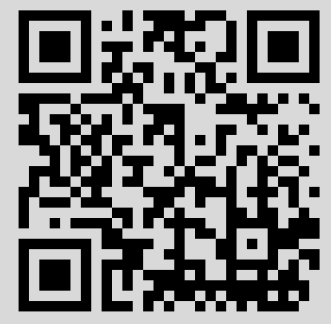


УДК 517.54

\section{СУММАТОРНЫЕ РАЦИОНАЛЬНЫЕ ОПЕРАТОРЫ ТИПА ДЖЕКСОНА}

\section{Е. А. Ровба}

Построены сумматорные рациональные положительные операторы типа Джексона $D_{4 n-n}(x ; f)$ на вещественной оси. Получена оценка приближений посредством таких операторов непрерывных на $\mathbb{R}$ функций $f$, имеющих конечные и равные между собой $\lim _{x \rightarrow-\infty} f(x)$ и $\lim _{x \rightarrow+\infty} f(x)$.

Библиография: 4 названия.

В настоящей заметке продолжены начатые в [1] исследования, посвященные построению положительных интерполящионных рациональных операторов.

Построены сумматорные операторы типа Джексона на вещественной оси и получена оценка скорости приближения функций $f \in C_{\infty}$. В полиномиальном случае такие операторы изучены достаточно полно (см., например, [2]). Интегральные рациональные операторы типа Джексона исследованы В. Н. Русаком [3].

Пусть $\left\{z_{k}\right\}_{k=1}^{+\infty}-$ последовательность комплексных чисел $z_{k}=\alpha_{k}+i \beta_{k}, \beta_{k}>0$, $k \in \mathbb{N}, z_{0}=i$. Рассмотрим синус-дробь Бернштейна $\sin \mu_{n}(x)$, где

$$
\mu_{n}(x)=\arg \left(z_{0}-x\right)+2 \sum_{k=1}^{n} \arg \left(z_{k}-x\right), \quad n \in \mathbb{N} .
$$

Функция $\sin \mu_{n}(x)$ имеет $2 n+1$ нулей на вещественной оси. Обозначим их через $x_{k}, k=$ $\overline{1,2 n+1}, \mu_{n}\left(x_{k}\right)=k \pi, k=\overline{1,2 n+1},-\infty<x_{1}<x_{2}<\cdots<x_{2 n}<x_{2 n+1}=\infty$.

Пусть задана функция $f \in C_{\infty}$, т.е. непрерывная на $\mathbb{R}$, и существуют конечные и равные между собой пределы

$$
\lim _{x \rightarrow-\infty} f(x) \quad \text { и } \quad \lim _{x \rightarrow+\infty} f(x) .
$$

Введем функцию

$$
D_{4 n-4}(x ; f)=\frac{1}{g_{n}(x)} \sum_{k=1}^{2 n+1} \frac{f\left(x_{k}\right)}{\mu_{n}^{\prime}\left(x_{k}\right)} G_{n}\left(x ; x_{k}\right), \quad x \in \mathbb{R},
$$


где

$$
\begin{gathered}
G_{n}\left(x ; x_{k}\right)=\frac{\left(1+x_{k}^{2}\right)\left\{\sin \left(\Phi_{n}(x)-\Phi_{n}\left(x_{k}\right)\right)\right\}^{4}}{\left(x-x_{k}\right)^{4}}, \quad k=\overline{1,2 n}, \\
\Phi_{n}(x)=\sum_{k=1}^{n} \arg \left(z_{k}-x\right), \quad n \in \mathbb{N}, \\
\frac{G_{n}\left(x ; x_{2 n+1}\right)}{\mu_{n}^{\prime}\left(x_{2 n+1}\right)}=\lim _{t \rightarrow+\infty} \frac{G_{n}(x ; t)}{\mu_{n}^{\prime}(t)}=\frac{\sin ^{4} \Phi_{n}(x)}{1+2 \sum_{k=1}^{n} \beta_{k}} .
\end{gathered}
$$

$\Phi$ ункцию $g_{n}(x)$ определим из условия, что $D_{4 n-4}(x ; 1) \equiv 1$, т.е.

$$
g_{n}(x)=\sum_{k=1}^{2 n+1} \frac{G_{n}\left(x ; x_{k}\right)}{\mu_{n}^{\prime}\left(x_{k}\right)} .
$$

Из $[4$, с. 115$]$ известно, что

$$
\sin \left(\Phi_{n}(x)-\Phi_{n}(t)\right)=\frac{1}{2 i} \frac{\prod_{k=1}^{n}\left(z_{k}-x\right)\left(\bar{z}_{k}-t\right)-\prod_{k=1}^{n}\left(\bar{z}_{k}-x\right)\left(z_{k}-t\right)}{\prod_{k=1}^{n}\left|z_{k}-x\right|\left|z_{k}-t\right|} .
$$

Тогда из (1) и (2) следует, что $D_{4 n-4}(x ; f)$ является рациональной функцией порядка не вьше $4 n-4$.

Лемма 1. Справедливы равенства

$$
\begin{gathered}
\sum_{k=1}^{n} \frac{\sin ^{2}\left(\Phi_{n}(x)-\Phi_{n}\left(x_{k}\right)\right)}{\mu_{n}^{\prime}\left(x_{k}\right)\left(x-x_{k}\right)^{2}}=\Phi_{n}^{\prime}(x), \\
g_{n}(x)=\frac{2}{3}\left(1+x^{2}\right)\left[\left(\Phi_{n}^{\prime}(x)\right)^{3}-\sum_{k=1}^{n} \frac{\beta_{k}^{3}}{\left(\left(x-\alpha_{k}\right)^{2}+\beta_{k}^{2}\right)^{3}}\right] \\
+\frac{1}{2} \sum_{k=1}^{n} \beta_{k} \frac{1+\alpha_{k}^{2}+\beta_{k}^{2}}{\left(\left(x-\alpha_{k}\right)^{2}+\beta_{k}^{2}\right)^{2}}, \quad n \in \mathbb{N} .
\end{gathered}
$$

ДокАЗАТЕЛЬСтво. Введем обозначение

$$
K_{n}(x ; t)=\frac{\sin ^{2}\left(\Phi_{n}(x)-\Phi_{n}(t)\right)}{(x-t)^{2}} .
$$

Покажем вначале, что

$$
\sum_{k=1}^{n} \frac{K_{n}\left(x ; x_{k}\right)}{\mu_{n}^{\prime}\left(x_{k}\right)}=\frac{1}{2 \pi i} \int_{\bar{\Gamma}} K(x ; t) \operatorname{ctg} \mu_{n}(t) d t,
$$

где $\Gamma$ - произвольный замкнутьй кусочно-гладкий непрерьвньй контур, содержащий внутри себя точки $z_{k}$ и $\bar{z}_{k}, k=\overline{0, n}$, и не содержащий внутри себя точек $x_{k}, k=\overline{1,2 n+1}$. 
Рассмотрим вначале подынтегральную функцию $K_{n}(x ; t) \operatorname{ctg} \mu_{n}(t)$ переменной $t$. Еcли учесть равенство (3), то нетрудно заметить, что во внешности контура $Г$ данная функция будет иметь простые полюсы в точках $x_{k}, k=\overline{1,2 n+1}$. Следовательно,

$$
\frac{1}{2 \pi i} \int_{\bar{\Gamma}} K_{n}(x ; t) \operatorname{ctg} \mu_{n}(t) d t=\sum_{k=1}^{2 n+1} \operatorname{res}_{t=x_{k}} K_{n}(x ; t) \operatorname{ctg} \mu_{n}(t) .
$$

Если $k=\overline{1,2 n}$, то

$$
\underset{t=x_{k}}{\operatorname{res}} K_{n}(x ; t) \operatorname{ctg} \mu_{n}(t)=K_{n}\left(x ; x_{k}\right) \frac{\cos \mu_{n}\left(x_{k}\right)}{\cos \mu_{n}\left(x_{k}\right) \mu_{n}^{\prime}\left(x_{k}\right)}=\left(\mu_{n}^{\prime}\left(x_{k}\right)\right)^{-1} K_{n}\left(x ; x_{k}\right) .
$$

Если же $k=2 n+1$, то нетрудно показать, что

$$
\begin{aligned}
\underset{t=x_{2 n+1}}{\operatorname{res}} K_{n}(x ; t) \operatorname{ctg} \mu_{n}(t) & =\lim _{t \rightarrow \infty} K_{n}(x ; t) \operatorname{ctg} \mu_{n}(t) \cdot t \\
& =\sin ^{2} \Phi_{n}(x) \lim _{t \rightarrow \infty} \frac{t}{(x-t)^{2}} \frac{\sin \mu_{n}(t)}{\cos \mu_{n}(t)}=\frac{\sin ^{2} \Phi_{n}(x)}{1+2 \sum_{k=1}^{n} \beta_{k}} .
\end{aligned}
$$

Таким образом, равенство (7) справедливо.

Покажем теперь, что $J_{n}=L_{n}, n \in \mathbb{N}$, где

$$
\begin{aligned}
J_{n} & =\frac{1}{2 \pi i} \int_{\bar{\Gamma}} K_{n}(x ; t) \operatorname{ctg} \mu_{n}(t) d t \quad \text { (интеграл из (7)), } \\
L_{n} & =\frac{1}{\pi} \int_{-\infty}^{+\infty} K_{n}(x ; t) d t .
\end{aligned}
$$

Действительно, учитывая равенства (6) и (3), нетрудно получить, что

$$
K(x ; t)=\frac{-1}{4(x-t)^{2}}\left(\frac{a_{n}(x) b_{n}(t)}{a_{n}(t) b_{n}(x)}-2+\frac{a_{n}(t) b_{n}(x)}{a_{n}(x) b_{n}(t)}\right),
$$

где

$$
\begin{gathered}
a_{n}(u)=\prod_{j=1}^{n}\left(z_{j}-u\right), \quad b_{n}(u)=\prod_{j=1}^{n}\left(\bar{z}_{j}-u\right), \\
\operatorname{ctg} \mu_{n}(t)=i \frac{\left(z_{0}-t\right) a_{n}^{2}(t)+\left(\bar{z}_{0}-t\right) b_{n}^{2}(t)}{\left(z_{0}-t\right) a_{n}^{2}(t)-\left(\bar{z}_{0}-t\right) b_{n}^{2}(t)}
\end{gathered}
$$

Следовательно, подьнтегральная функция интеграла $J_{n}$ имеет внутри Г простые полюсы в точках $t=z_{k}$ и $t=\bar{z}_{k}, k=\overline{1, n}$. Значит,

$$
J_{n}=-\sum_{k=1}^{n}\left(\underset{t=z_{k}}{\operatorname{res}} K_{n}(x ; t) \operatorname{ctg} \mu_{n}(t)+\underset{t=\bar{z}_{k}}{\operatorname{res}} K_{n}(x ; t) \operatorname{ctg} \mu_{n}(t)\right)
$$

И

$$
\underset{t=z}{\operatorname{res}} K_{n}(x ; t) \operatorname{ctg} \mu_{n}(t)=\lim _{t \rightarrow z} K_{n}(x ; t) \operatorname{ctg} \mu_{n}(t)(t-z), \quad z=z_{k}, \quad z=\bar{z}_{k} .
$$


Легко убедиться, что $\mu_{n}\left(z_{k}\right)=-i, \mu_{n}\left(\bar{z}_{k}\right)=i, k=\overline{1, n}$ (см. (10)).

Следовательно,

$$
\begin{aligned}
& \underset{t=z_{k}}{\operatorname{res}} K_{n}(x ; t) \operatorname{ctg} \mu_{n}(t)=-i \underset{t=z_{k}}{\operatorname{res}} K_{n}(x ; t), \\
& \underset{t=\bar{z}_{k}}{\operatorname{res}} K_{n}(x ; t) \operatorname{ctg} \mu_{n}(t)=i \underset{t=\bar{z}_{k}}{\operatorname{res}} K_{n}(x ; t), \quad k=\overline{1, n},
\end{aligned}
$$

и

$$
J_{n}=i \sum_{k=1}^{n}\left(\operatorname{res}_{t=z_{k}} K_{n}(x ; t)-\underset{t=\bar{z}_{k}}{\operatorname{res}} K_{n}(x ; t)\right) .
$$

Выражение справа в этом равенстве есть не что иное, как интеграл $L_{n}($ см. (8)), представленньй через вычеты относительно особьх точек, лежащих как в верхней, так и в нижней полуплоскостях. Таким образом, действительно, $J_{n}=L_{n}, n \in \mathbb{N}$. Но интеграл $L_{n}$ вычислен в $[4$, с. 115$]$ :

$$
L_{n}=\Phi_{n}^{\prime}(x), \quad \Phi_{n}^{\prime}(x)=\sum_{k=1}^{n} \frac{\beta_{k}}{\left(\alpha_{k}-x\right)^{2}+\beta_{k}^{2}} .
$$

Следовательно, равенство (4) имеет место.

Справедливость соотношения (5) устанавливается по такой же схеме. Вначале установим, что $g_{n}(x)=J_{n}^{*}($ см. $(2))$, где

$$
J_{n}^{*}=\frac{1}{2 \pi i} \int_{\bar{\Gamma}} G_{n}(x ; t) \operatorname{ctg} \mu_{n}(t) d t
$$

$\Gamma$ - тот же контур, что и в интеграле (7). Затем докажем, что $J_{n}^{*}=L_{n}^{*}$, где

$$
L_{n}^{*}=\frac{1}{\pi} \int_{-\infty}^{+\infty} G_{n}(x ; t) d t .
$$

Останется воспользоваться леммой из $[4$, с. 40$]$ о вычислении интеграла $L_{n}^{*}$, где показьвается, что $L_{n}^{*}$ равен выражению, стоящему справа в соотношении (5).

Равенство интегралов $J_{n}^{*}$ и $L_{n}^{*}$ устанавливается аналогично приведенному вьше доказательству соотношения $J_{n}=L_{n}$.

Обозначив через $\varkappa_{n}(t)=a_{n}(t) / b_{n}(t)($ см. $(9))$, будем иметь

$$
\begin{aligned}
\frac{G_{n}(x ; t)}{1+t^{2}}= & \frac{1}{16} \varkappa_{n}^{2}(x) \varkappa_{n}^{-2}(t)-\frac{1}{4} \varkappa_{n}(x) \varkappa_{n}^{-1}(t) \\
& +\frac{3}{8}-\frac{1}{4} \varkappa_{n}^{-1}(x) \varkappa(t)+\frac{1}{16} \varkappa_{n}^{-2}(x) \varkappa_{n}^{2}(t) .
\end{aligned}
$$

Функция $G_{n}(x ; t) \operatorname{ctg} \mu_{n}(t)$ вне $Г$ имеет простые полюсы в точках $x_{k}, k=\overline{1,2 n+1}$, и внутри $\Gamma$ - полюсы второго порядка в точках $z_{k}, \bar{z}_{k}, k=\overline{1, n}$.

Следовательно,

$$
J_{n}^{*}=\sum_{k=1}^{2 n+1} \operatorname{res}_{t=x_{k}} G_{n}(x ; t) \operatorname{ctg} \mu_{n}(t)=\sum_{k=1}^{2 n+1} \frac{G_{n}(x ; t)}{\mu_{n}^{\prime}\left(x_{k}\right)}=g_{n}(x) .
$$


Итак, $g_{n}(x)=J_{n}^{*}$. С другой стороны,

$$
\begin{aligned}
& J_{n}^{*}=-\sum_{k=1}^{2 n+1}\left(\underset{t=z_{k}}{\operatorname{res}} G_{n}(x ; t) \operatorname{ctg} \mu_{n}(t)+\underset{t=\bar{z}_{k}}{\operatorname{res}} G_{n}(x ; t) \operatorname{ctg} \mu_{n}(t)\right) \\
& \underset{t=z_{k}, \bar{z}_{k}}{\operatorname{res}} G_{n}(x ; t) \operatorname{ctg} \mu_{n}(t)=\lim _{t \rightarrow z_{k}, \bar{z}_{k}} \frac{\partial}{\partial t}\left(G_{n}(x ; t) \operatorname{ctg} \mu_{n}(t)\left(t-z_{k}\right)^{2}\right) \\
& =\lim _{t \rightarrow z_{k}, \bar{z}_{k}}\left[\frac{\partial}{\partial t}\left(G_{n}(x ; t)\left(t-z_{k}\right)^{2}\right) \operatorname{ctg} \mu_{n}(t)\right. \\
& \left.+G_{n}(x ; t)\left(t-z_{k}\right)^{2} \frac{d}{d t} \operatorname{ctg} \mu_{n}(t)\right] \text {. }
\end{aligned}
$$

Нетрудно вычислить, что (см. (10))

$$
\left.\frac{d}{d t} \operatorname{ctg} \mu_{n}(t)\right|_{t=z_{k}, \bar{z}_{k}}=0, \quad k=\overline{1, n}
$$

Следовательно,

$$
\begin{aligned}
& \underset{t=z_{k}}{\operatorname{res}}\left(G_{n}(x ; t) \operatorname{ctg} \mu_{n}(t)\right)=-i \underset{t=z_{k}}{\operatorname{res}} G_{n}(x ; t), \\
& \underset{t=\bar{z}_{k}}{\operatorname{res}}\left(G_{n}(x ; t) \operatorname{ctg} \mu_{n}(t)\right)=i \underset{t=\bar{z}_{k}}{\operatorname{res}} G_{n}(x ; t) .
\end{aligned}
$$

Подставив эти выражения в (12), получим

$$
J_{n}^{*}=i \sum_{k=1}^{2 n+1}\left(\underset{t=z_{k}}{\operatorname{res}} G_{n}(x ; t)-\underset{t=\bar{z}_{k}}{\operatorname{res}} G_{n}(x ; t)\right) .
$$

А это есть не что иное, как интеграл $L_{n}^{*}($ см. (11)). Учитывая вышесказанное, доказательство леммы 1 завершено.

ЛЕмма 2 [4, с. 135]. Справедлива оченка

$$
g_{n}(x)>\frac{1+x^{2}}{2}\left(\Phi_{n}^{\prime}(x)\right)^{3}, \quad x \in \mathbb{R}
$$

ТЕоремА. Если $f \in C_{\infty}$, то имеет место неравенство

$$
\left|f(x)-D_{4 n-4}(x ; f)\right| \leqslant 2 \omega\left(\frac{\sqrt{2}}{\left(1+x^{2}\right) \Phi_{n}^{\prime}(x)}\right), \quad x \in \mathbb{R}
$$

где $\omega(\delta)$ - модуль непрерывности функиии $F(t)=f(\operatorname{tg}(t / 2))$. 
ДоКАЗАТЕЛЬСТво. Исходя из соотношения (1) и (2), имеем

$$
f(x)-D_{4 n-4}(x ; f)=\frac{1}{g_{n}(x)} \sum_{k=1}^{2 n+1} \frac{f(x)-f\left(x_{k}\right)}{\mu_{n}\left(x_{k}\right)} G_{n}\left(x ; x_{k}\right) .
$$

Пусть $x=\operatorname{tg}(t / 2), x_{k}=\operatorname{tg} t_{k} / 2, k=\overline{1,2 n+1}\left(t_{2 n+1}=\pi\right)$.

Полагаем также $\delta_{n}(x)=\sqrt{2}\left(\left(1+x^{2}\right) \Phi_{n}^{\prime}(x)\right)^{-1}$ (см. (1)). Фиксируем произвольное $x \in \mathbb{R}$ и обозначим через

$$
\begin{aligned}
\Omega_{t} & =\left\{k: k=\overline{1,2 n+1},\left|t-t_{k}\right|<\delta_{n}(x),\left|t-t_{k} \pm 2 \pi\right|<\delta_{n}(x)\right\}, \\
C \Omega_{t} & =\{1,2, \ldots, 2 n+1\} \backslash \Omega_{t} .
\end{aligned}
$$

Если $\delta_{n}(x) \geqslant 2 \pi$, то множество $C \Omega_{t}$ является пустьм. В дальнейшем, не ограничивая обшности, будем считать, что $\delta_{n}(x)<2 \pi$ и множество $C \Omega_{t}$ не является пустьм.

Тогда, исходя из равенства (13), будем иметь

$$
\begin{aligned}
\left|f(x)-D_{4 n-4}(x ; f)\right| \leqslant & \frac{1}{g_{n}(x)} \sum_{k \in \Omega_{t}} \frac{\left|F(t)-F\left(t_{k}\right)\right|}{\mu_{n}^{\prime}\left(x_{k}\right)} G_{n}\left(x ; x_{k}\right) \\
& +\frac{1}{g_{n}(x)} \sum_{k \in C \Omega_{t}} \frac{\left|F(t)-F\left(t_{k}\right)\right|}{\mu_{n}^{\prime}\left(x_{k}\right)} G_{n}\left(x ; x_{k}\right) .
\end{aligned}
$$

Очевидно, что

$$
\left|F(t)-F\left(t_{k}\right)\right| \leqslant \omega\left(\delta_{n}(x)\right), \quad k \in \Omega_{t} .
$$

Если же $k \in C \Omega_{t}$, то нетрудно показать, что

$$
\left|F(t)-F\left(t_{k}\right)\right| \leqslant \omega\left(\gamma\left(t, t_{k}\right)\right),
$$

где

$$
\gamma\left(t, t_{k}\right)= \begin{cases}\left|t-t_{k}\right|, & \text { если }\left|t-t_{k}\right| \leqslant \pi, \\ 2 \pi-\left|t-t_{k}\right|, & \text { если } \pi<\left|t-t_{k}\right|<2 \pi .\end{cases}
$$

Подставляя эти оценки в (14), получим

$$
\begin{aligned}
\left|f(x)-D_{4 n-4}(x ; f)\right| & \leqslant \frac{\omega\left(\delta_{n}(x)\right)}{g_{n}(x)} \sum_{k \in \Omega_{t}} \frac{G_{n}\left(x ; x_{k}\right)}{\mu_{n}^{\prime}\left(x_{k}\right)}+\frac{1}{g_{n}(x)} \sum_{k \in C \Omega_{t}} \frac{\omega\left(\gamma\left(t, t_{k}\right)\right)}{\mu_{n}^{\prime}\left(x_{k}\right)} G_{n}\left(x ; x_{k}\right) \\
& =\omega\left(\delta_{n}(x)\right) s_{1}(x)+s_{2}(x),
\end{aligned}
$$

где

$$
\begin{aligned}
& s_{1}(x)=\frac{1}{g_{n}(x)} \sum_{k \in \Omega_{t}} \frac{G_{n}\left(x ; x_{k}\right)}{\mu_{n}^{\prime}\left(x_{k}\right)}, \\
& s_{2}(x)=\frac{1}{g_{n}(x)} \sum_{k \in C \Omega_{t}} \frac{\omega\left(\gamma\left(t, t_{k}\right)\right)}{\mu_{n}^{\prime}\left(x_{k}\right)} G_{n}\left(x ; x_{k}\right) .
\end{aligned}
$$


Оценим вначале сумму $s_{2}(x)$. Воспользовавшись неравенством

$$
\omega\left(\gamma\left(t, t_{k}\right)\right) \leqslant\left(\gamma\left(t, t_{k}\right) \delta_{n}^{-1}(x)+1\right) \omega\left(\delta_{n}(x)\right),
$$

получим

$$
s_{2}(x) \leqslant \frac{\delta_{n}^{-1}(x)}{g_{n}(x)} \sum_{k \in C \Omega_{t}} \frac{\gamma\left(t, t_{k}\right)}{\mu_{n}^{\prime}\left(x_{k}\right)} G_{n}\left(x ; x_{k}\right) \omega\left(\delta_{n}(x)\right)+\frac{1}{g_{n}(x)} \sum_{k \in C \Omega_{t}} \frac{G_{n}\left(x ; x_{k}\right)}{\mu_{n}^{\prime}\left(x_{k}\right)} \omega\left(\delta_{n}(x)\right) .
$$

Учитьвая равенство (2), имеем

$$
s_{1}(x)+\frac{1}{g_{n}(x)} \sum_{k \in C \Omega_{t}} \frac{G_{n}\left(x ; x_{k}\right)}{\mu_{n}^{\prime}\left(x_{k}\right)}=I
$$

Остается оценить первое слагаемое в правой части неравенства (17). Пусть

$$
s_{2}^{(1)}(x)=\frac{\delta_{n}^{-1}(x)}{g_{n}(x)} \sum_{k \in C \Omega_{t}} \frac{\gamma\left(t, t_{k}\right)}{\mu_{n}^{\prime}\left(x_{k}\right)} G_{n}\left(x ; x_{k}\right) .
$$

Рассмотрим следующую функцию:

$$
\zeta\left(x ; x_{k}\right)=\gamma\left(t ; t_{k}\right) \frac{\sqrt{1+x_{k}^{2}} \sqrt{1+x^{2}}}{\left|x-x_{k}\right|}, \quad k \in C \Omega_{t}
$$

Учитьвая, что $x=\operatorname{tg}(t / 2), x_{k}=\operatorname{tg}\left(t_{k} / 2\right)$, и воспользовавшись неравенством $\sin u \geqslant u$, $u \in[0 ; \pi / 2]$, получим (см. также $(15))$

$$
\zeta\left(x ; x_{k}\right)=\frac{\gamma\left(t ; t_{k}\right)}{\sin \left(\left|t-t_{k}\right| / 2\right)} \leqslant \pi, \quad k \in C \Omega_{t} .
$$

Исходя из этого неравенства, найдем следующую оценку суммы (19):

$$
s_{2}^{(1)}(x) \leqslant \frac{\delta_{n}^{-1}(x)}{\sqrt{1+x^{2}} g_{n}(x)} \sum_{k \in C \Omega_{t}} \frac{\sqrt{1+x_{k}^{2}}}{\mu_{n}^{\prime}\left(x_{k}\right)}\left|\frac{\sin \left(\Phi_{n}(x)-\Phi_{n}\left(x_{k}\right)\right)}{x-x_{k}}\right|^{3}
$$

Теперь применим неравенство Коши-Буняковского

$$
\begin{aligned}
s_{2}^{(1)}(x) & \leqslant \frac{\delta_{n}^{-1}(x)}{\sqrt{1+x^{2}} g_{n}(x)} \sum_{k \in C \Omega_{t}} \frac{1}{\sqrt{\mu_{n}^{\prime}\left(x_{k}\right)}}\left|\frac{\sin \left(\Phi_{n}(x)-\Phi_{n}\left(x_{k}\right)\right)}{x-x_{k}}\right| \frac{\sqrt{1+x_{k}^{2}} K_{n}\left(x ; x_{k}\right)}{\sqrt{\mu_{n}^{\prime}\left(x_{k}\right)}} \\
& \leqslant \frac{\delta_{n}^{-1}(x)}{\sqrt{1+x^{2}} g_{n}(x)}\left(\sum_{k=1}^{2 n+1} \frac{K_{n}\left(x ; x_{k}\right)}{\mu_{n}^{\prime}\left(x_{k}\right)}\right)^{1 / 2}\left(\sum_{k=1}^{2 n+1} \frac{G_{n}\left(x ; x_{k}\right)}{\mu_{n}^{\prime}\left(x_{k}\right)}\right)^{1 / 2} \cdot
\end{aligned}
$$


Остается воспользоваться соотношениями (2) и (4)

$$
\begin{aligned}
s_{2}^{(1)}(x) & \leqslant \frac{\delta_{n}^{-1}(x)}{\sqrt{1+x^{2}} g_{n}(x)}\left(\left(1+x^{2}\right) \Phi_{n}^{\prime}(x)\right)^{1 / 2}\left(g_{n}(x)\right)^{1 / 2} \\
& =\frac{\sqrt{\Phi_{n}(x)}}{\delta_{n}(x) \sqrt{g_{n}(x)}}=\left(\frac{\left(1+x_{k}^{2}\right)\left(\Phi_{n}^{\prime}(x)\right)^{3}}{2 g_{n}(x)}\right)^{1 / 2}
\end{aligned}
$$

и применить лемму 2 , чтобы получить неравенство

$$
s_{2}^{(1)}(x) \leqslant I .
$$

В совокупности соотношения (16)-(18) и (20) позволяют заключить:

$$
\left|f(x)-D_{4 n-4}(x ; f)\right| \leqslant 2 \omega\left(\delta_{n}(x)\right), \quad x \in \mathbb{R},
$$

что и завершает доказательство теоремы.

Гродненский государственный университет

\section{СПИСОК ЦИТИРОВАННОЙ ЛИТЕРАТУРЫ}

[1] Ровба Е. А. Интерполяционные рациональные операторы типа Фейера и Валле-Пуссена // Матем. заметки. 1993. Т. 53. № 2. С. 114-121.

[2] Бугаец В. П., Мартынюк В.Т. Точная константа приближения непрерывных функций сумматорными операторами типа Джексона // Укр. матем. ж. 1977. Т. 29. №6. С. 791-796.

[3] Русак В.Н. О порядке приближения положительньми рациональньми операторами // Изв. АН БССР. Сер. физ.-матем. наук. 1975. № 3. С. 39-46.

[4] Русак В.Н. Рациональные функции как аппарат приближения. Минск: Изд-во БГУ, 1979. 